\title{
A versatile platform for single-molecule enzymology of restriction endonuclease
}

\author{
Xin Wang*, Jingyuan $\mathrm{Nie}^{\dagger}$, Yi Li*, Hai Pan*, Peng Zheng ${ }^{\dagger}$,***, \\ Meng Qin ${ }^{\dagger, \S, * *}$, Yi Cao, ${ }^{*, \boldsymbol{\Phi}, * *}$ and Wei Wang, ${ }^{*, * * *}$ \\ *National Laboratory of Solid State Microstructure \\ School of Physics, Nanjing University 22 Hankou Road \\ Nanjing, Jiangsu 210093, P. R. China \\ ${ }^{\dagger}$ School of Chemistry and Chemical Engineering \\ Nanjing University 163 Xianlin Avenue \\ Nanjing, Jiangsu 210023, P. R. China \\ tpengz@nju.edu.cn \\ §qinmeng@nju.edu.cn \\ "caoyi@nju.edu.cn \\ "wangwei@nju.edu.cn
}

Received 23 April 2018

Accepted 30 May 2018

Published 10 July 2018

\begin{abstract}
Enzymes are the major players for many biological processes. Fundamental studies of the enzymatic activity at the single-molecule level provides important information that is otherwise inaccessible at the ensemble level. Yet, these single-molecule experiments are technically difficult and generally require complicated experimental design. Here, we develop a Holliday junction (HJ)-based platform to study the activity of restriction endonucleases at the single-molecule level using single-molecule FRET (sm-FRET). We show that the intrinsic dynamics of HJ can be used as the reporter for both the enzyme-binding and the substrate-release events. Thanks to the multiple-arms structure of HJ, the fluorophore-labeled arms can be different from the surface anchoring arm and the substrate arm. Therefore, it is possible to independently change the substrate arm to study different enzymes with similar functions. Such a design is extremely useful for the systematic study of enzymes from the same family or enzymes bearing different pathologic mutations. Moreover, this method can be easily extended to study other types of DNA-binding enzymes without too much modification of the design. We anticipate it can find broad applications in single-molecule enzymology.
\end{abstract}

Keywords: Single molecule; Förster resonance energy transfer; enzymology; kinetics; holliday junction.

\footnotetext{
${ }^{* *}$ Corresponding authors.
}

This is an Open Access article published by World Scientific Publishing Company. It is distributed under the terms of the Creative Commons Attribution 4.0 (CC-BY) License. Further distribution of this work is permitted, provided the original work is properly cited. 


\section{Wang et al.}

\section{Introduction}

Enzymes, generally proteins or few ribonucleic acids, are macromolecular biological catalysts which play irreplaceable roles in almost every physiological activity in vivo. Many natural and artificial enzymes are developed for the acceleration of chemical reactions in the synthesis industry. ${ }^{1-3}$ For example, some complicated drugs can be produced by one-pot enzymatic synthesis in vitro. ${ }^{4}$ Enzymes are also important tools for molecular biology, bioengineering and materials science. Restriction enzymes and DNA ligases are indispensable tools for recombinant DNA techniques. Cas9 involved in CRISPR system is now the star for genome engineering, ${ }^{5,6}$ gene knockout or knockdown, ${ }^{7}$ transcriptional activation ${ }^{8}$ and cancer research. ${ }^{9-11}$ Many enzymes are used to construct hydrogels, ${ }^{12-14}$ catalysts ${ }^{15,16}$ and drug delivery systems. ${ }^{17-19}$ In all these applications, understanding the action mechanism of enzymes is critical, which lays the foundation for the improvement of their performances. On the other hand, the change of activities of enzymes in vivo due to mutations is tightly related with many diseases. ${ }^{20}$ Fundamental enzymology studies have a strong impact on medicine and healthcare. Many techniques, such as stopped-flow system ${ }^{21,22}$ and continuous-flow system, ${ }^{23,24}$ have been used to study enzymology at the bulk level.

Single-molecule studies can provide many insights of enzymes that are otherwise inaccessible from bulk

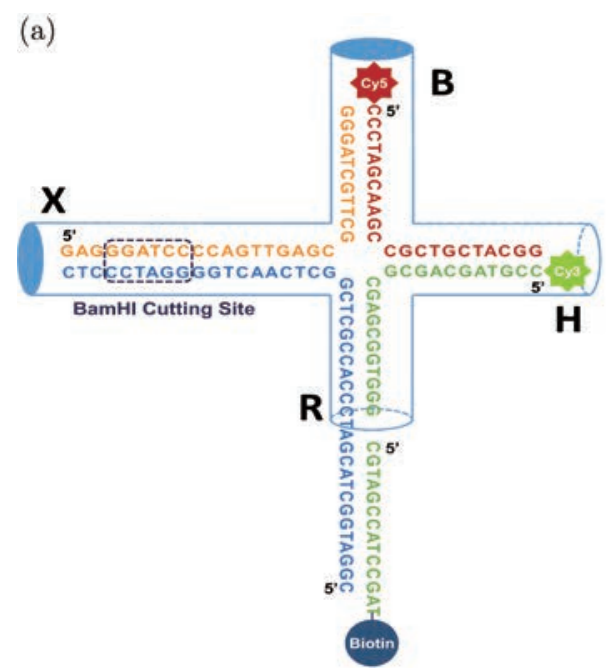

studies. Currently, single-molecule techniques have been proven to be powerful tools for studying folding dynamics of proteins ${ }^{25-27}$ and DNA, ${ }^{28-30}$ biomolecular interaction ${ }^{31-33}$ and enzymatic reaction dynamics. $^{34-37}$ Specifically, single-molecule Förster resonance energy transfer (sm-FRET) is widely applied for studying the structure, dynamics and function of proteins at the single-molecule level in real-time. ${ }^{38-41}$ In typical sm-FRET studies, a pair of donor-acceptor fluorescent dyes is introduced to suitable positions of the protein or nucleic acid structure. The distance change of the two labeled positions can be monitored by the FRET effect of the two fluorophores with nanometer resolution in situ, making it a perfect tool for enzymology. ${ }^{41-44}$ However, these studies relied either on the specific labeling of enzyme and the substrate using fluorescent dyes $^{38-44}$ or on the intrinsic fluorescence of the products. $^{45}$ The former method needs tedious fluorophore labeling and is technically difficult to implement to different systems. The latter one is limited to only a few enzyme systems. In addition, the labeling of fluorescent dyes might affect the intrinsic dynamic of an enzyme. Moreover, in many enzymological processes, the structural changes are too small to be detected even with the state-of-theart sm-FRET techniques. Herein, we present a versatile platform to measure the activity of DNA endonuclease, based on Holliday junction (HJ) [Fig. 1(a)]. HJ comprises four arms namely X, B, R and $\mathrm{H}$, with two stable native conformations: The

(b)

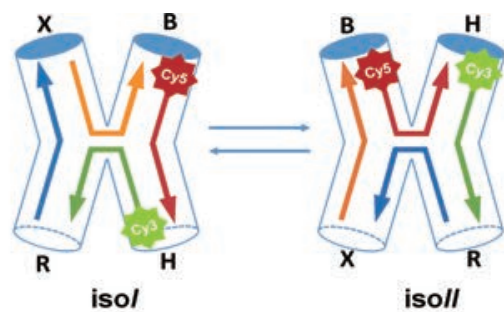

(c)

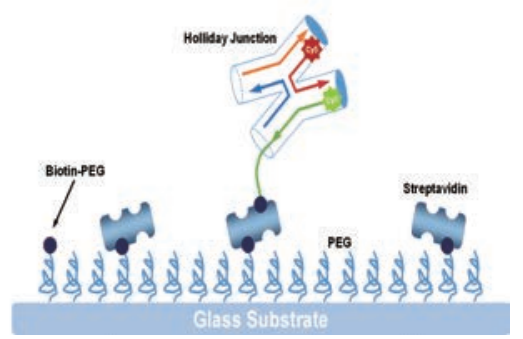

Fig. 1. (Color online) (a) The scheme of designed HJ. The b-strand (red), x-strand (orange), r-strand (blue), h-strand and biotin strand (both green) form the HJ with four arms (B, H, R and X). (b) The scheme of "isoI" and "isoII" states. The "isoI" state has longer distance between Cy3 and Cy5 than "isoII" state. (c) Surface immobilization strategy for sm-FRET experiments. The components are not drawn to relative scale. 
"isoI" state with the B-arm far from the $\mathrm{H}$-arm and the "isoII" state with the B-arm close to the $\mathrm{H}$-arm [Fig. 1(b)]. ${ }^{46}$ The intrinsic dynamics of HJ has been extensively studied by sm-FRET and theory. ${ }^{46-49}$ We use the dynamics of HJ as the reporter for the enzymatic reactions taking place on the $\mathrm{X}$-arm. We hypothesize that binding of an enzyme to the X-arm and subsequent enzymatic reactions can affect the intrinsic dynamics of HJ, thus can be detected by analyzing the dynamics of HJ. A similar method was used to study protein-DNA interaction previously by Sarkar et al. ${ }^{50}$ In this method, the fluorescent dyes are labeled on the reporter arms (H- and R-arms) of HJ instead of the enzyme or the substrate directly, which minimizes the side-effect of dye labeling on the dynamics and activity of the enzyme. Moreover, the enzyme-binding arm (X-arm) and the surface-immobilizing arm (R-arm) are decoupled with the reporter arms, providing plenty of opportunities for the studies of various DNA-binding enzymes. In principle, this method can be used to quickly measure the activity of different enzymes without the need to change the fluorescent substrates. Using restriction enzymes as the example, enzyme binding may slow down the intrinsic dynamics of $\mathrm{HJ}$ due to the stabilizing of the two native states. However, after the enzymatic reaction, the $\mathrm{X}$-arm is cut and becomes shorter, leading to faster conversions between "isoI" and "isoII" states. Therefore, the substrate binding and catalytic activity can both be measured from the change of the dynamics of HJ. We have experimentally proved this idea using BamHI as the representative restriction endonuclease [Fig. 1(c)].

\section{Method}

\subsection{The preparation of $\mathrm{HJ}$}

HJ-b-strand (5'-Cy5-CCCTAGCAAGCCGCTGCTACGG) and HJ-h-strand (5'-Cy3-CCGTAGCAGCGCGAGCGGTGGG) were purchased from Invitrogen (ThermoFisher Scientific, Inc., USA). HJ-x-strand (5'-GAGGGATCCCCAGTTGAGCGCTTGCTAGGG), HJ-r-strand (5'-CGGATGGCTACGATCCCACCGCTCGGCTCAACTGGGGATCCCTC) and biotin strand (5'-CGTAGCCATCCGAT-Biotin) were purchased from GenScript (Nanjing, China). All the b-, h-, x-, h- and biotinstrands were dissolved in TN buffer $(50 \mathrm{mM}$ Tris, $50 \mathrm{mM} \mathrm{NaCl}, \mathrm{pH}=8.0$ ), respectively. Then, five kinds of strands were mixed in $\mathrm{TN}$ buffer to reach the final concentration of $1 \mu \mathrm{M}$ and the mixture was slowly annealed by PCR machine (slowly cooled from $95^{\circ} \mathrm{C}$ to $15^{\circ} \mathrm{C}$, then raised to $65^{\circ} \mathrm{C}$ and dropped to $25^{\circ} \mathrm{C}$ for three cycles and finally cooled to $4^{\circ} \mathrm{C}$ ). The purity of $\mathrm{HJ}$ was confirmed by native-PAGE.

\subsection{The digestion of $\mathrm{HJ}$}

The mixture of HJ and BamHI (R0136S, New England BioLabs, USA) was incubated at $37^{\circ} \mathrm{C}$ for $1 \mathrm{~h}$ and then analyzed by the denatured PAGE followed by silver staining.

\subsection{Modification of the glass coverslip}

The scheme of coverslip modification is shown in Fig. 1(c). Coverslip was biotinylated following previous reports. ${ }^{51-53}$ The $0.15-\mathrm{mm}$ glass coverslip $\left(24 \times 40 \mathrm{~mm}^{2}\right.$, Micro Cover Glasses No. 1, VWR International, LLC) was cleaned in piranha solution $\left(98 \% \mathrm{H}_{2} \mathrm{SO}_{4}: 30 \% \mathrm{H}_{2} \mathrm{O}_{2}=7: 3, \mathrm{v} / \mathrm{v}\right)$ for $30 \mathrm{~min}$ to be hydroxylated. After thoroughly rinsing with MilliQwater, the coverslip was dried under argon airflow. Then we gently heat the coverslip using blast alcohol burner to decompose any possible fluorescent impurities. After cooling to room temperature, the hydroxyl-functional coverslip was immersed in acetone solution containing $10 \%$ (3-aminopropyl) triethoxysilane (APTES, Sigma-Aldrich, USA) for $30 \mathrm{~min}$ and rinsed thoroughly with acetone and MilliQ-water, respectively. After being dried under argon airflow, the amino-functional coverslip was PEGylated by immersing in aqueous solution $(\mathrm{pH}=8.0)$ containing $100-\mathrm{mM} \mathrm{NaHCO} 3,15 \mathrm{mg} /$ mL Biotin-PEG-SVA (MW: 5 kDa, Laysan Bio, Inc., USA) and $150 \mathrm{mg} / \mathrm{mL}$ Methyl-PEG-SVA (MW: $5 \mathrm{kDa}$, Laysan Bio, Inc., USA) for at least $3 \mathrm{~h}$. Finally, the coverslip was rinsed thoroughly with MilliQ-water, followed by drying under argon airflow. The biotin-functional coverslip should be kept in dark and be used freshly.

\subsection{Sample cell for sm-FRET experiments}

The biotin-functional coverslip was made into a sandwich structure cell with several sample channels. TN buffer containing $1 \%$ Tween $20(\mathrm{v} / \mathrm{v})$ was 
added and incubated for $20 \mathrm{~min}$, and then was rinsed with TN buffer three times. TN buffer containing $200 \mu \mathrm{g} / \mathrm{mL}$ streptavidin was added to each sample channel and incubated for $1 \mathrm{~min}$. After rinsing with TN buffer three times, TN buffer containing $10-50 \mathrm{pM}$ HJ was injected into the sample channel and incubated for 20 min then rinsed with TN buffer three times.

\subsection{Sm-FRET experiments}

The single-molecule FRET experiments were executed on an Olympus IX-71 with an oil immersion UAPON $100 \times$ OTIRF objective lens (numerical aperture $=1.49$, Olympus). Cy3 was excited by a 532-nm laser and the emission fluorescence of $\mathrm{Cy} 3$ and that of $\mathrm{Cy} 5$ were split into two channels by a dichroic filter (FF640-FDi01, Semrock). The emission fluorescence of two channels passed through two band-pass filters (FF01-585/40 and FF01-67567 , Semrock), respectively, and the final fluorescence signals were collected by an electron-multiplying charge-coupled device camera (IXon897, Andor Technology). The sm-FRET experiments were carried out in Tris buffer $(50 \mathrm{mM}$ Tris, $\mathrm{pH}=8.0$ ) containing $50 \mathrm{mM} \mathrm{MgCl}_{2}$. About $1-\mu \mathrm{L}$ BamHI (20 units) was diluted in $100-\mu \mathrm{L}$ Tris buffer (50 mM Tris, $50 \mathrm{mM} \mathrm{MaCl}_{2}, \mathrm{pH}=8.0$ ) for digestion in sm-FRET experiments. Dynamics of the digested BamHI-HJ was obtained 20 min later after an addition of BamHI. Here 0.8\% (w/v) glucose, 1-mg/ $\mathrm{mL}$ glucose oxidase, $0.04-\mathrm{mg} / \mathrm{mL}$ catalase and 2 $\mathrm{mM}$ Trolox were involved as oxygen scavenger system. ${ }^{54,55}$

\section{Results and Discussion}

\subsection{Intrinsic dynamics of HJ with the BamHI restriction site (BamHI-HJ)}

We first used sm-FRET to study the intrinsic dynamics of the newly designed BamHI-HJ containing five strands and longer X- and R-arms. A typical trace of sm-FRET is shown in Fig. 2(a). Cy3 signal (green) and Cy5 signal (red) hopped stochastically between the states of low or high intensity. The efficiency of energy transfer Ewas calculated by Eq. (1):

$$
E=I_{\mathrm{Cy} 5} /\left(I_{\mathrm{Cy} 3}+I_{\mathrm{Cy} 5}\right),
$$

where $I_{\mathrm{Cy} 3}$ and $I_{\mathrm{Cy} 5}$ were the signal intensities of $\mathrm{Cy} 3$ and $\mathrm{Cy} 5$, respectively. The transfer efficiency depends on the distance between $\mathrm{Cy} 3$ and $\mathrm{Cy} 5$, according to Eq. (2):

$$
E=1 /\left(1+\left(r / R_{0}\right)^{6}\right),
$$

where $r$ is the distance between $\mathrm{Cy} 3$ and $\mathrm{Cy} 5$ and $R_{0}$ is the characteristic distance at which the sm-FRET efficiency is 0.5. As shown in Fig. 2(b), the transfer efficiency also switched at two conformational states. As the HJ changed to "isoI" state, Cy3 was separated from Cy5. As a result, the transfer efficiency dropped to lower state. Later, the HJ jumped back to "isoII" state, corresponding to higher transfer efficiency [Fig. 2(b)]. The collected traces were summed up to make a histogram. The histogram of sm-FRET efficiency shows a clear bimodal distribution [Fig. 2(c)]. The distribution was fitted by a double-Gaussian function and gave one state located at 0.23 and another state located at 0.71 . These results confirmed that introducing the BamHI restriction site on $\mathrm{HJ}$ did not significantly change its conformational dynamics. ${ }^{46}$ The dwell time on each state was also measured [Figs. 2(d) and 2(e)]. The exponential fittings (dark red) gave the transition rates of $0.107 \mathrm{~s}^{-1}$ and $0.085 \mathrm{~s}^{-1}$, for "isoI" to "isoII" and "isoII" to "isoI", respectively. The dynamics is summarized in Table 1. Both transition rates were much slower than the original HJ, with shorter X- and R-arms as reported by previous work. ${ }^{46}$ This also indicated that the dynamics of HJ is sensitive to the length of each arm. As a result, digesting the restriction site by BamHI should increase the dynamics of HJ.

\subsection{Dynamics of the digested BamHI-HJ}

Before the sm-FRET experiments, we examined the efficiency of BamHI digestion in bulk using the PAGE gel. We constructed a complex of r-strand and x-strand following the similar protocol for HJ. This complex and BamHI-HJ were incubated with BamHI, respectively, for $1 \mathrm{~h}$ before being analyzed using denatured PAGE. As shown in Fig. 3(c), both the digested BamHI-HJ (lane 4) and the digested complex (lane 6) had one additional band (red box), compared to the original BamHI-HJ (lane 5) and the complex (lane 7). This confirmed that the extended X-arm in BamHI-HJ can be effectively digested by BamHI. Next, we studied the dynamics of digested BamHI-HJ using sm-FRET. A typical FRET trace is shown in Fig. 3(a). The transfer 

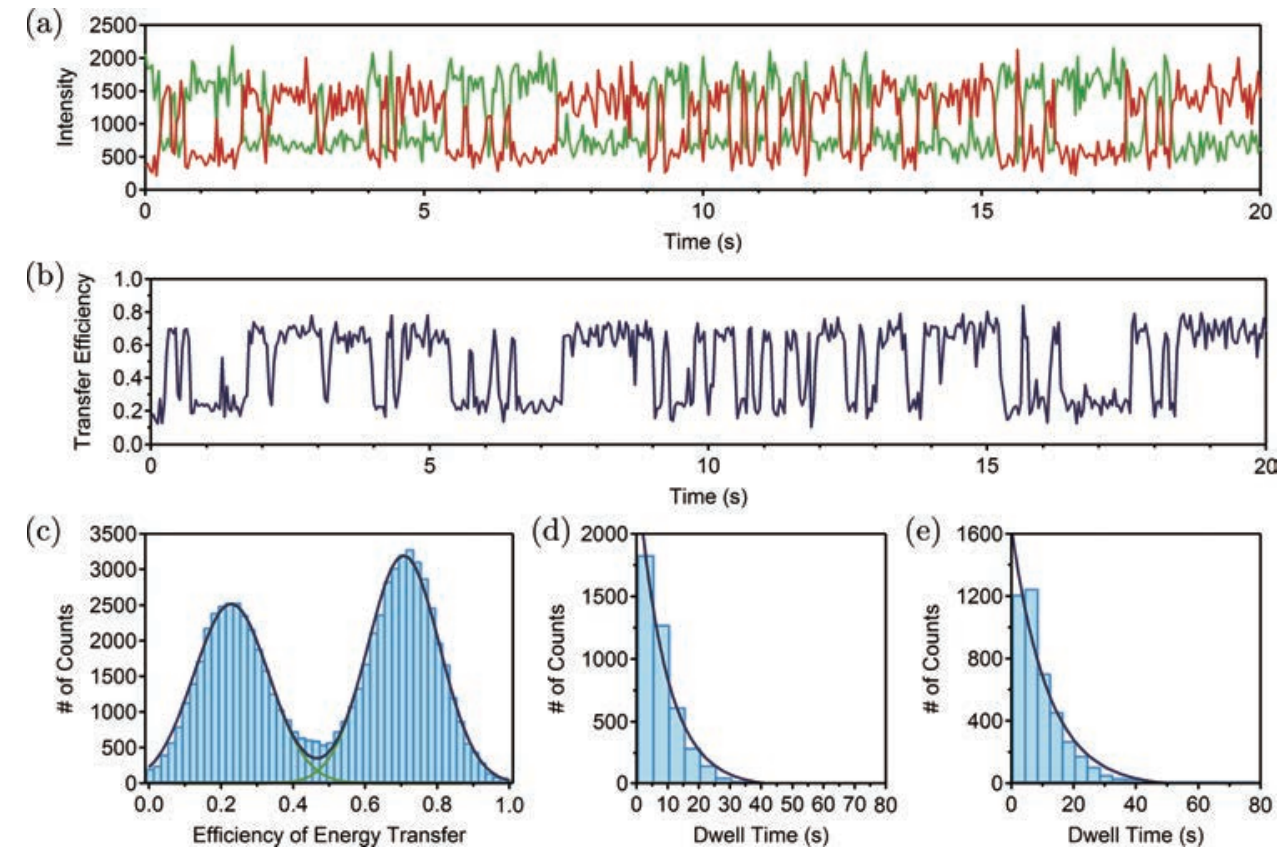

Fig. 2. (Color online) (a) Typical traces of sm-FRET experiments. Green and red traces refer to Cy3 and Cy5 signals, respectively. (b) The dependence of efficiency of energy transfer on time. (c) The efficiency of energy transfer distribution of BamHI-HJ. Green curves are Gaussian fittings and dark blue curve is the sum of green curves. (d) The histogram for the dwell time at the "isoII" state. Dark blue curve is exponential fitting. (e) The histogram for the dwell time at the "isoI" state. Dark blue curve is exponential fitting. The data in (a)-(e) were collected in the absence of BamHI.

efficiency hopped more quickly with shorter dwell time after BamHI digestion of the X-arm [Fig. 3(b)]. The distribution of transfer efficiency is also a clear bimodal distribution [Fig. 3(d)] and the doubleGaussian fitting yielded the efficiencies of 0.24 for "isoI" state and 0.68 for "isoII" state. The transfer efficiency of each state did not change much, compared to that of each state before digestion. The duration time of each state was used to create the histograms. The exponential fitting yielded the transfer rates of $0.160 \mathrm{~s}^{-1}$ and $0.125 \mathrm{~s}^{-1}$, for "isoI" to "isoII" and "isoII" to "isoI", respectively [Figs. 3(e) and 3(f)]. The dynamics is summarized in Table 1. Note that the transfer rates for "isoI" to "isoII" and

Table 1. Results of dynamics.

\begin{tabular}{lccc}
\hline & & $\begin{array}{c}\text { Transfer } \\
\text { efficiency }\end{array}$ & $\begin{array}{c}\text { Transition } \\
\text { rate }\left(\mathrm{s}^{-1}\right)\end{array}$ \\
\hline BamHI-HJ & isoI & 0.23 & 0.107 \\
& isoII & 0.71 & 0.085 \\
Digested BamHI-HJ & $i s o I$ & 0.24 & 0.160 \\
& $i s o I I$ & 0.68 & 0.125 \\
Original HJ $^{\mathrm{a}}$ & $i s o I$ & 0.2 & 5.7 \\
& isoII & 0.6 & 6.1 \\
\hline
\end{tabular}

${ }^{a}$ Data from a previous work of McKinney et al. ${ }^{46}$
"isoII" to "isoI" increased by $50 \%$ and $47 \%$, respectively, after BamHI digestion, suggesting that the dynamics of $\mathrm{HJ}$ can indeed serve as a reporter for the action of BamHI.

\subsection{Direct observation of BamHI digestion based on the HJ dynamics}

As mentioned above, the transition rates of the two states both rose after the BamHI digestion. Statistically, the turnover between the two states will become faster. Thus, we define the average turnover frequency, $f$, which is equal to the average number of turnovers per second in few seconds, as a measurable quantity to distinguish the undigested and digested BamHI-HJ. As shown in Figs. 4(a) and $4(\mathrm{~b})$, the signal jumped from one to another more frequently after BamHI was added to the system at about $16 \mathrm{~s}$ (marked by orange arrow). We calculated the average turnover frequency in a time span of $10 \mathrm{~s}$. The frequency-time curves were fitted with the Boltzmann function shown in Eq. (3):

$$
f=A_{2}+\left(A_{1}-A_{2}\right) /\left(1+\exp \left(\left(t-t_{0}\right) / d t\right)\right),
$$

where $f$ is the average turnover frequency as defined above, $t$ is the time, $A_{1}, A_{2}, t_{0}$ and $d t$ are fitting 
(a)

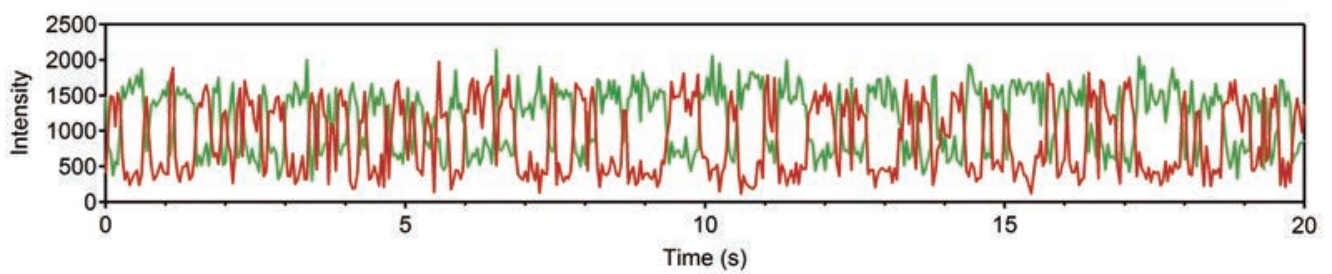

(b)

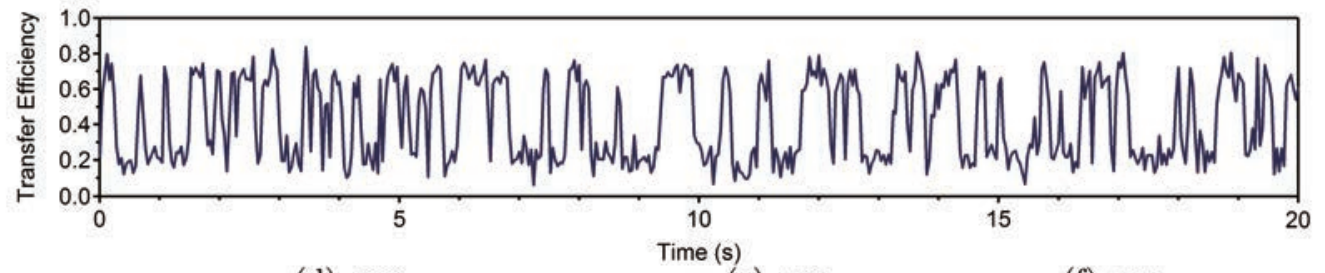

(c)
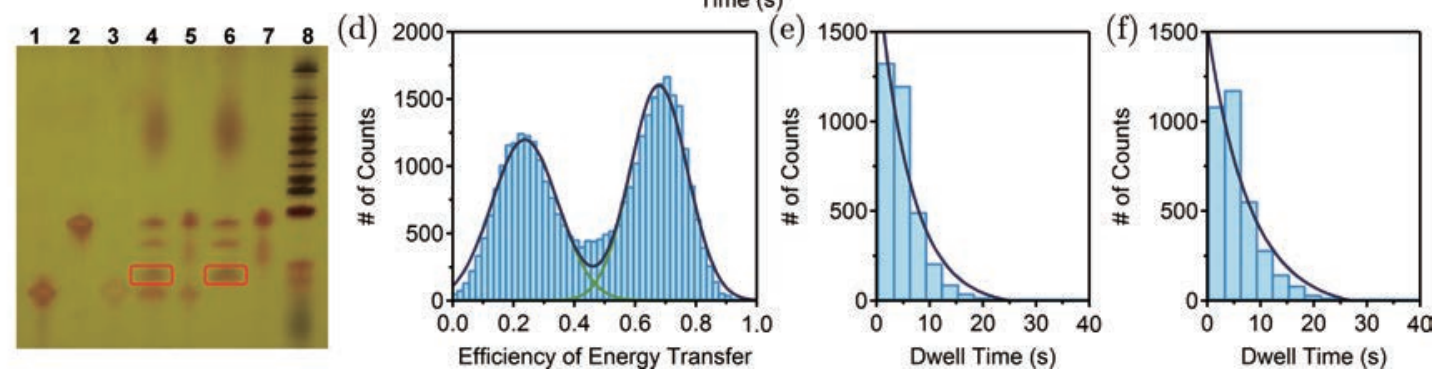

Fig. 3. (Color online) (a) Typical traces of digested BamHI-HJ. Green and red traces refer to Cy3 and Cy5 signals, respectively. (b) The time trajectory of the FRET efficiency. (c) The denatured PAGE results of BamHI digestion of HJ. Lane 1: h-strand, lane 2: r-strand, lane 3: biotin strand, lane 4: BamHI-HJ digested by BamHI, lane 5: BamHI-HJ only, lane 6: the complex of r-strand and x-strand digested by BamHI, lane 7: complex of r-strand and x-strand only and lane 8: low molecular weight DNA ladder (N3233L, New England BioLabs). (d) The efficiency of energy transfer distribution of BamHI-HJ after BamHI digestion. Green curves are Gaussian fittings to the two peaks and dark blue curve is the sum of the two Gaussian fitting curves. (e) The histogram for the dwell time at the "isoII" state. Dark blue curve is the exponential fitting. (f) The histogram for the dwell time at the "isoI" state. Dark blue curve is exponential fitting. The data in panels (a), (b) and (d)-(f) were collected after the digestion of BamHI.

parameters. The time interval $\Delta t$ for the enzyme to finish the digestion reaction equals four times of $d t$ based on Eq. (3) [Fig. 4(g)]. Note that, although $f$ showed relatively large intrinsic fluctuations due to the non-Gaussian distribution (exponential) of the dwell time, such intrinsic fluctuations did not affect the two-state fitting too much. This is because the enzyme kinetics of BamHI is much slower than the dynamics of HJ. Moreover, the transition of $f$ from the low state to the high state of each trace did not occur at the same time after the addition of BamHI [Figs. $4(\mathrm{c})-4(\mathrm{f})]$. This lag time $\left(\tau_{\text {lag }}\right.$, time interval from adding enzyme to the midpoint of $\Delta t$, which is just the parameter $t_{0}$ ) suggested the heterogeneous nature of the enzymatic reaction at the singlemolecule level and corresponded to the combined time for an enzyme to bind to the restriction site on the X-arm and then successfully digest the substrate. The distributions of $\Delta t$ and $\tau_{\text {lag }}$ are summarized in Figs. 4(h) and 4(i), respectively. Considering the enzyme substrate exists in three different states: unbound $(U)$, bound $(B)$ and cut $(C)$, the kinetic relations among these states can be described as follows:

$$
[U] \stackrel{k_{1}}{\rightarrow}[B] \stackrel{k_{2}}{\rightarrow}[C]
$$

where $k_{1}$ and $k_{2}$ refer to the binding rate and the digestion rate, respectively. The unbinding of enzyme from the substrate was ignored as we did not observe unbinding events (causing the increase of turnover frequency from the low state to the original state). For single-molecular turnover events, the probability, $p(\Delta t)$, is defined as follows ${ }^{56}$ :

$$
p(\Delta t)=k_{2} \exp \left(-k_{2} \Delta t\right) .
$$

Fitting Eq. (5) to the experimental data yields $k_{2}$ of $0.23 \mathrm{~s}^{-1}$, indicating that once binding on the restriction site, BamHI has a $90 \%$ probability to finish cutting in $10 \mathrm{~s}$.

As the lag time, $\tau_{\text {lag }}$, is the combined time for binding and digestion processes, the probability of 

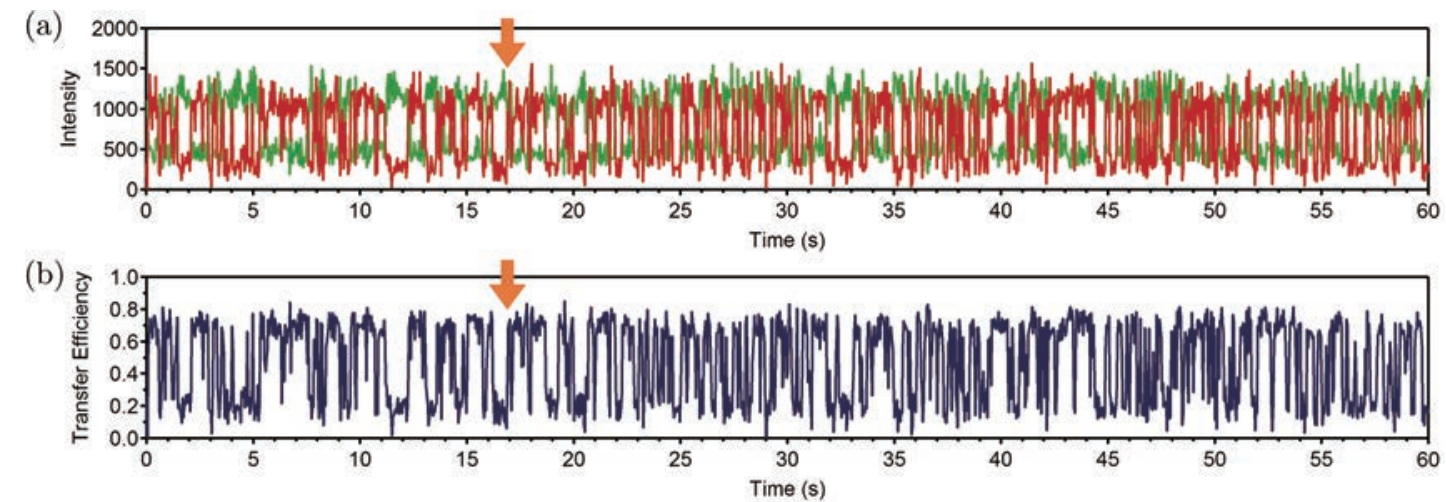

(c)

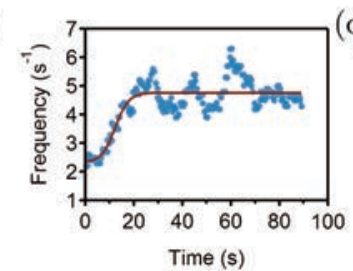

(g)

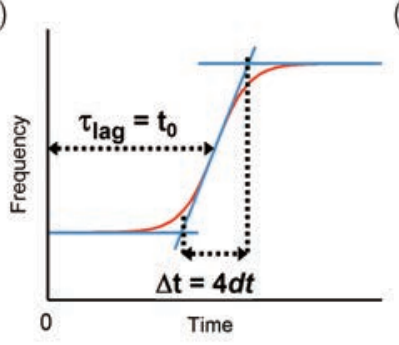

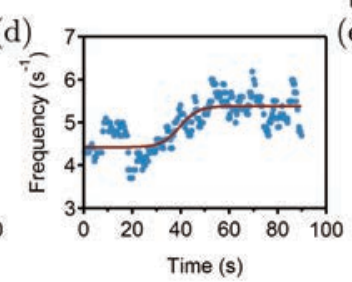

(h)

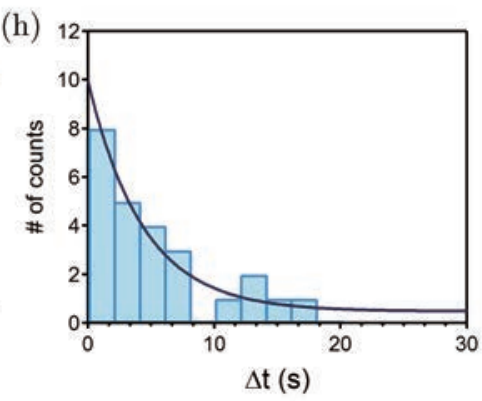

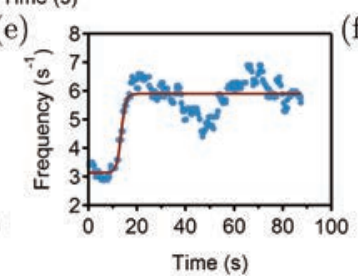

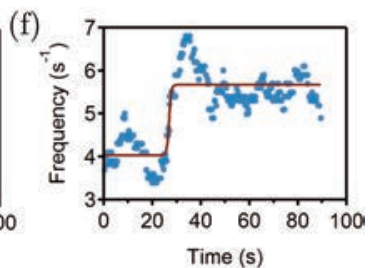

(i)

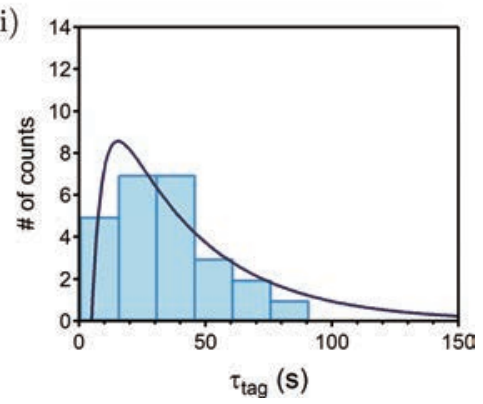

Fig. 4. (Color online) (a) Typical trace and (b) transfer efficiency during the BamHI digestion process. The orange arrow shows the moment when BamHI is added. (c)-(f) Four representative traces of the turnover frequency. BamHI was added at time 0. (g) Fitting scheme of panels (c)-(f). Here $\Delta t$ is the time interval for the enzyme to finish the digestion reaction and $\tau_{\text {lag }}$ is the time needed from the addition of BamHI to the midpoint of $\Delta t$. (h) Histogram of the time span for each transition $(\Delta t)$. Dark blue curve is exponential fitting. (i) The distribution of the lag time $\left(\tau_{\text {lag }}\right)$. Dark blue curve is the fitting to Eq. (6).

$\tau_{\text {lag }}$ for the serial events at the single-molecule level can be described as shown in Eq. (6) (Ref. 56):

$$
p\left(\tau_{\text {lag }}\right)=k_{1} k_{2}\left(e^{-k_{1} \tau_{\text {lag }}}-e^{-k_{2} \tau_{\operatorname{lag}}}\right) /\left(k_{2}-k_{1}\right) .
$$

Fitting Eq. (6) to the data with fixed $k_{2}$ of $0.230 \mathrm{~s}^{-1}$ yielded $k_{1}$ of $0.0286 \mathrm{~s}^{-1}$, which is nearly one-fold slower than $k_{2}$ [Fig. 4(i)]. This is probably due to the low concentration of BamHI used in the reaction condition. These experiments demonstrated the successful measurement of kinetics of BamHI digestion at the single-molecule level using the dynamics of HJ as the reporter.

\section{Conclusion}

In summary, we developed a HJ-based platform to study the activity of restriction endonucleases at the single-molecule level using single-molecule
FRET. This method allows the activity of different restriction endonucleases to be measured without extra work to change the fluorescent-labeled enzymes or substrates. Using BamHI as the representative example, we showed that both the enzyme-binding and the substrate-release events can be observed based on the distinct dynamics of $\mathrm{HJ}$ at the corresponding states. Since this new tool greatly simplifies the single-molecule studies of DNA-binding enzymes, we anticipate that it can be broadly applied to study more complicated and important enzymes and provides unprecedented information for the understanding of their functions.

\section{Conflict of Interest}

We have no conflict interest to declare. 


\section{Acknowledgments}

Xin Wang and Jingyuan Nie contributed equally to this work. The authors greatly appreciate the financial support from National Natural Science Foundation of China (Grant Nos. 21522402, 11674153, 11374148, 11334004 and 21771103), the Fundamental Research Funds for the Central Universities (Nos. 020414380070, 020414380050 and 020414380058), Natural Science Foundation of Jiangsu Province (No. BK20160639) and the Shuangchuang Program of Jiangsu Province.

\section{References}

1. M. Ohashi, F. Liu, Y. Hai, M. Chen, M. C. Tang, Z. Yang, M. Sato, K. Watanabe, K. N. Houk, Y. Tang, "SAM-dependent enzyme-catalysed pericyclic reactions in natural product biosynthesis," Nature 549, 502-506 (2017).

2. J. Latham, E. Brandenburger, S. A. Shepherd, B. R. K. Menon, J. Micklefield, "Development of halogenase enzymes for use in synthesis," Chem. Rev. 118, 232-269 (2018).

3. M. Winkler, M. Geier, S. P. Hanlon, B. Nidetzky, A. Glieder, "Human enzymes for organic synthesis," Angew. Chem., Int. Ed. Engl. (2018), doi: 10.1002/ anie. 201800678.

4. S. Meng, W. Han, J. Zhao, X. H. Jian, H. X. Pan, G. L. Tang, "A six-oxidase cascade for tandem C-H bond activation revealed by reconstitution of bicyclomycin biosynthesis," Angew. Chem., Int. Ed. Engl. 57, 719-723 (2018).

5. P. Mali, L. Yang, K. M. Esvelt, J. Aach, M. Guell, J. E. DiCarlo, J. E. Norville, G. M. Church, "RNAguided human genome engineering via Cas9," Science 339, 823-826 (2013).

6. L. Cong, F. A. Ran, D. Cox, S. Lin, R. Barretto, N. Habib, P. D. Hsu, X. Wu, W. Jiang, L. A. Marraffini, F. Zhang, "Multiplex genome engineering using CRISPR/Cas systems," Science 339, 819-823 (2013).

7. J. Joung, S. Konermann, J. S. Gootenberg, O. O. Abudayyeh, R. J. Platt, M. D. Brigham, N. E. Sanjana, F. Zhang, "Genome-scale CRISPR-Cas9 knockout and transcriptional activation screening," Nat. Protoc. 12, 828-863 (2017).

8. S. Konermann, M. D. Brigham, A. E. Trevino, J. Joung, O. O. Abudayyeh, C. Barcena, P. D. Hsu, N. Habib, J. S. Gootenberg, H. Nishimasu, O. Nureki, F. Zhang, "Genome-scale transcriptional activation by an engineered CRISPR-Cas9 complex," Nature 517, 583-588 (2015).

9. W. Xue, S. Chen, H. Yin, T. Tammela, T. Papagiannakopoulos, N. S. Joshi, W. Cai, G. Yang, R.
Bronson, D. G. Crowley, F. Zhang, D. G. Anderson, P. A. Sharp, T. Jacks, "CRISPR-mediated direct mutation of cancer genes in the mouse liver," Nature 514, 380-384 (2014).

10. S. Chen, N. E. Sanjana, K. Zheng, O. Shalem, K. Lee, X. Shi, D. A. Scott, J. Song, J. Q. Pan, R. Weissleder, H. Lee, F. Zhang, P. A. Sharp, "Genome-wide CRISPR screen in a mouse model of tumor growth and metastasis," Cell 160, 1246-1260 (2015).

11. R. J. Platt, S. Chen, Y. Zhou, M. J. Yim, L. Swiech, H. R. Kempton, J. E. Dahlman, O. Parnas, T. M. Eisenhaure, M. Jovanovic, D. B. Graham, S. Jhunjhunwala, M. Heidenreich, R. J. Xavier, R. Langer, D. G. Anderson, N. Hacohen, A. Regev, G. Feng, P. A. Sharp, F. Zhang, "CRISPR-Cas9 knockin mice for genome editing and cancer modeling," Cell 159, 440-455 (2014).

12. N. Rauner, M. Meuris, M. Zoric, J. C. Tiller, "Enzymatic mineralization generates ultrastiff and tough hydrogels with tunable mechanics," Nature 543, 407-410 (2017).

13. S. H. Um, J. B. Lee, N. Park, S. Y. Kwon, C. C. Umbach, D. Luo, "Enzyme-catalysed assembly of DNA hydrogel," Nat. Mater. 5, 797-801 (2006).

14. O. B. Ayyub, P. Kofinas, "Enzyme induced stiffening of nanoparticle-hydrogel composites with structural color," ACS Nano 9, 8004-8011 (2015).

15. H.-P. M. De Hoog, I. W. C. E. Arends, A. E. Rowan, J. J. L. M. Cornelissen, R. J. M. Nolte, "A hydrogelbased enzyme-loaded polymersome reactor," Nanoscale 2, 709-716 (2010).

16. K. V. Nguyen, Y. Holade, S. D. Minteer, "DNA redox hydrogels: Improving mediated enzymatic bioelectrocatalysis," ACS Catal. 6, 2603-2607 (2016).

17. K. J. C. van Bommel, M. C. A. Stuart, B. L. Feringa, J. van Esch, "Two-stage enzyme mediated drug release from LMWG hydrogels," Org. Biomol. Chem. 3, 2917-2920 (2005).

18. P. D. Thornton, R. J. Mart, S. J. Webb, R. V. Ulijn, "Enzyme-responsive hydrogel particles for the controlled release of proteins: Designing peptide actuators to match payload," Soft Mat. 4, 821-827 (2008).

19. P. D. Thornton, R. J. Mart, R. V. Ulijn, "Enzymeresponsive polymer hydrogel particles for controlled release," Adv. Mater. 19, 1252-1256 (2007).

20. J. Liao, C.-X. Wu, C. Burlak, S. Zhang, H. Sahm, M. Wang, Z.-Y. Zhang, K. W. Vogel, M. Federici, S. M. Riddle, R. J. Nichols, D. Liu, M. R. Cookson, T. A. Stone, Q. Q. Hoang, "Parkinson diseaseassociated mutation R1441H in LRRK2 prolongs the "active state" of its GTPase domain," Proc. Natl. Acad. Sci. USA 111, 4055-4060 (2014).

21. A. M. Caccuri, G. Antonini, P. G. Board, M. W. Parker, M. Nicotra, M. Lo Bello, G. Federici, G. Ricci, "Proton release on binding of glutathione to 
alpha, mu and delta class glutathione transferases," Biochem. J. 344, 419-425 (1999).

22. F. S. Chang, P. C. Chen, R. L. Chen, F. M. Lu, T. J. Cheng, "Real-time assay of immobilized tannase with a stopped-flow conductometric device," Bioelectrochemistry 69, 113-116 (2006).

23. X. Zhou, R. Medhekar, M. D. Toney, "A continuousflow system for high-precision kinetics using small volumes," Anal. Chem. 75, 3681-3687 (2003).

24. J. Zhang, A. E. Cass, "Kinetic study of site directed and randomly immobilized his-tag alkaline phosphatase by flow injection chemiluminescence," $J$. Mol. Recognit. 19, 243-246 (2006).

25. H. Chen, G. Yuan, R. S. Winardhi, M. Yao, I. Popa, J. M. Fernandez, J. Yan, "Dynamics of equilibrium folding and unfolding transitions of titin immunoglobulin domain under constant forces," J. Am. Chem. Soc. 137, 3540-3546 (2015).

26. H. Chen, X. Zhu, P. Cong, M. P. Sheetz, F. Nakamura, J. Yan, "Differential mechanical stability of filamin A rod segments," Biophys. J. 101, 1231-1237 (2011).

27. C. Lv, X. Gao, W. Li, B. Xue, M. Qin, L. D. Burtnick, H. Zhou, Y. Cao, R. C. Robinson, W. Wang, "Singlemolecule force spectroscopy reveals force-enhanced binding of calcium ions by gelsolin," Nat. Commun. 5, 4623 (2014).

28. Y. Sun, W. Di, Y. Li, W. Huang, X. Wang, M. Qin, W. Wang, Y. Cao, " $\mathrm{Mg}^{2+}$-dependent high mechanical anisotropy of three-way-junction pRNA as revealed by single-molecule force spectroscopy," Angew. Chem., Int. Ed. Engl. 56, 9376-9380 (2017).

29. M. de Messieres, J. C. Chang, B. Brawn-Cinani, A. La Porta, "Single-molecule study of G-quadruplex disruption using dynamic force spectroscopy," Phys. Rev. Lett. 109, 058101 (2012).

30. X. M. Hou, Y. B. Fu, W. Q. Wu, L. Wang, F. Y. Teng, P. Xie, P. Y. Wang, X. G. Xi, "Involvement of G-triplex and G-hairpin in the multi-pathway folding of human telomeric G-quadruplex," Nucleic Acids Res. 45, 11401-11412 (2017).

31. A. Bhattacharjee, Y. Wang, J. Diao, C. M. Price, "Dynamic DNA binding, junction recognition and G4 melting activity underlie the telomeric and genome-wide roles of human CST," Nucleic Acids Res. 45, 12311-12324 (2017).

32. S. W. Stahl, M. A. Nash, D. B. Fried, M. Slutzki, Y. Barak, E. A. Bayer, H. E. Gaub, "Single-molecule dissection of the high-affinity cohesin-dockerin complex," Proc. Natl. Acad. Sci. USA 109, 2043120436 (2012).

33. W. Huang, M. Qin, Y. Li, Y. Cao, W. Wang, "Dimerization of cell-adhesion molecules can increase their binding strength," Langmuir 33, 1398-1404 (2017).

34. P. Kosuri, J. Alegre-Cebollada, J. Feng, A. Kaplan, A. Ingles-Prieto, C. L. Badilla, B. R. Stockwell,
J. M. Sanchez-Ruiz, A. Holmgren, J. M. Fernandez, "Protein folding drives disulfide formation," Cell 151, 794-806 (2012).

35. D. Singh, Y. Wang, J. Mallon, O. Yang, J. Fei, A. Poddar, D. Ceylan, S. Bailey, T. Ha, "Mechanisms of improved specificity of engineered Cas9s revealed by single-molecule FRET analysis," Nat. Struct. Mol. Biol. 25, 347-354 (2018).

36. M. K. Nahas, T. J. Wilson, S. Hohng, K. Jarvie, D. M. Lilley, T. Ha, "Observation of internal cleavage and ligation reactions of a ribozyme," Nat. Struct. Mol. Biol. 11, 1107-1113 (2004).

37. M. Sorokina, H.-R. Koh, S. S. Patel, T. Ha, "Fluorescent Lifetime trajectories of a single fluorophore reveal reaction intermediates during transcription initiation," J. Am. Chem. Soc. 131, 9630-9631 (2009).

38. X. Zhuang, L. E. Bartley, H. P. Babcock, R. Russell, T. Ha, D. Herschlag, S. Chu, "A single-molecule study of RNA catalysis and folding," Science $\mathbf{2 8 8}$, 2048-2051 (2000).

39. S. Myong, M. M. Bruno, A. M. Pyle, T. Ha, "Springloaded mechanism of DNA unwinding by hepatitis C virus NS3 helicase," Science 317, 513-516 (2007).

40. T. Mori, R. D. Vale, M. Tomishige, "How kinesin waits between steps," Nature 450, 750-754 (2007).

41. W. Lin, J. Ma, D. Nong, C. Xu, B. Zhang, J. Li, Q. Jia, S. Dou, F. Ye, X. Xi, Y. Lu, M. Li, "Helicase stepping investigated with one-nucleotide resolution fluorescence resonance energy transfer," Phys. Rev. Lett. 119, 138102 (2017).

42. M. Dyla, D. S. Terry, M. Kjaergaard, T. L. Sorensen, J. L. Andersen, J. P. Andersen, C. R. Knudsen, R. B. Altman, P. Nissen, S. C. Blanchard, "Dynamics of P-type ATPase transport revealed by single-molecule FRET," Nature 551, 346-351 (2017).

43. M. Lu, H. P. Lu, "Revealing multiple pathways in T4 lysozyme substep conformational motions by single-molecule enzymology and modeling," J. Phys. Chem. B 121, 5017-5024 (2017).

44. S. Ticau, L. J. Friedman, N. A. Ivica, J. Gelles, S. P. Bell, "Single-molecule studies of origin licensing reveal mechanisms ensuring bidirectional helicase loading," Cell 161, 513-525 (2015).

45. H. P. Lu, L. Xun, X. S. Xie, "Single-molecule enzymatic dynamics," Science 282, 1877-1882 (1998).

46. S. A. McKinney, A. C. Declais, D. M. Lilley, T. Ha, "Structural dynamics of individual Holliday junctions," Nat. Struct. Biol. 10, 93-97 (2003).

47. S. Hohng, C. Joo, T. Ha, "Single-molecule threecolor FRET," Biophys. J. 87, 1328-1337 (2004).

48. S. A. McKinney, A. D. J. Freeman, D. M. J. Lilley, T. Ha, "Observing spontaneous branch migration of Holliday junctions one step at a time," Proc. Natl. Acad. Sci. USA 102, 5715-5720 (2005). 
X. Wang et al.

49. C. Hyeon, J. Lee, J. Yoon, S. Hohng, D. Thirumalai, "Hidden complexity in the isomerization dynamics of Holliday junctions," Nat. Chem. 4, 907-914 (2012).

50. S. K. Sarkar, N. M. Andoy, J. J. Benitez, P. R. Chen, J. S. Kong, C. He, P. Chen, "Engineered Holliday junctions as single-molecule reporters for protein-DNA interactions with application to a MerR-family regulator," J. Am. Chem. Soc. 129, 12461-12467 (2007).

51. R. Roy, S. Hohng, T. Ha, "A practical guide to singlemolecule FRET," Nat. Methods 5, 507-516 (2008).

52. C. Chen, B. Stevens, J. Kaur, D. Cabral, H. Liu, Y. Wang, H. Zhang, G. Rosenblum, Z. Smilansky, Y. E. Goldman, B. S. Cooperman, "Single-molecule fluorescence measurements of ribosomal translocation dynamics," Mol. Cell 42, 367-377 (2011).

53. S. D. Chandradoss, A. C. Haagsma, Y. K. Lee, J.-H. Hwang, J.-M. Nam, C. Joo, "Surface passivation for single-molecule protein studies," J. Vis. Exp. (2014), doi: $10.3791 / 50549$.

54. R. E. Benesch, R. Benesch, "Enzymatic removal of oxygen for polarography and related methods," Science 118, 447-448 (1953).

55. I. Rasnik, S. A. McKinney, T. Ha, "Nonblinking and long-lasting single-molecule fluorescence imaging," Nat. Methods 3, 891-893 (2006).

56. X. S. Xie, H. P. Lu, "Single-molecule enzymology," J. Biol. Chem. 274, 15967-15970 (1999). 